\title{
Conscious sedation: considering operator and patient safety
}

Richard Harris

Head of Compliance

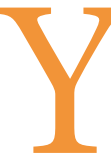

ou have a duty to ensure that a patient's right to adequate anxiety control as part of their care and treatment is provided

if necessary. For many patients, this will involve conscious sedation at a level whereby the patient remains conscious and is able to both understand and respond to verbal commands.

Operator safety sometimes takes a backseat to patient safety, although both are equally important.

The practice owner must provide a safe working clinical environment for staff, with the necessary equipment and be large enough to allow the operator, support staff and the patient to move around freely. This included space for emergency procedures to be performed.

During sedation, patients are exposed to relatively high concentrations of anaesthetic gases for a short period. But the operator and staff may well be exposed to lower concentrations, but for longer periods or more frequently, normally from the patient's exhaled breath, so checking for leaks around the breathing circuit and facemask is essential.

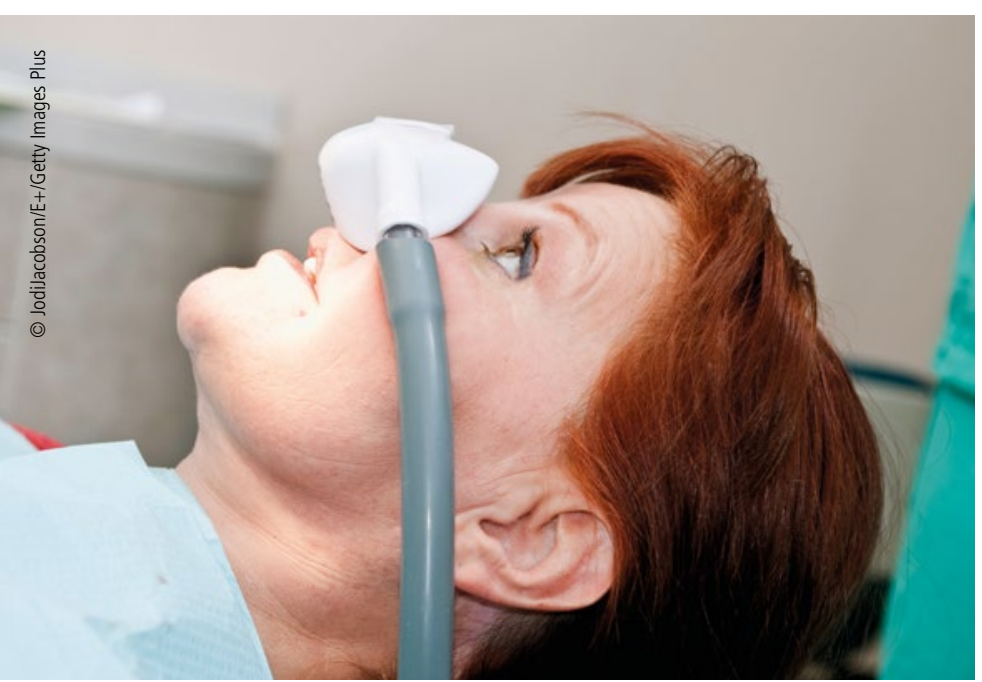

The potential effects of exposure to waste anaesthetic gases are nausea, dizziness, headaches and fatigue mixed with more physical impairments, such as reduced fertility and problems during pregnancy.

The HSE advises on workplace exposure limits for anaesthetic gases (nitrous oxide, halothane, enflurane and isoflurane) and monitoring exposure is normally undertaken by measuring the presence of the anaesthetic gas in the breathing zone where sedation is provided. Biological monitoring can also complement air monitoring when air sampling techniques alone may not give a reliable indication of exposure. For dentistry, this involves analysis of breath and/or blood samples. It reflects the total uptake of a chemical, for example via inhalation, ingestion, and/or through the skin.

Because of these issues, you should have a scavenging system to remove waste anaesthetic gases from the operating room and this should exhaust to an area where the waste gases will not be reintroduced into the clinical environment. The operating room should be well-ventilated to ensure regular changes of air which also helps disperse medical gases rapidly.

All team members must be adequately trained and have the knowledge to ensure safe and effective delivery of the sedation, including the use of equipment, general safety procedures and what emergency action to take. Their clinical skills are supported by validated education and training, and a minimum of 12 hours of verifiable CPD every five years. Good communication in the team is essential for the safety of all and policies and protocols should be written clearly avoid potential misunderstanding. Every team member should know their role and what is expected of them and feel confident to ask questions if unsure about anything. They should be aware of any potential adverse events.

The equipment must be purpose-designed and conform to accepted standards, being installed by a specialist company and thereafter validated and verified You must keep records of all maintenance and servicing undertaken.

Warning and alarm systems allow the monitoring of equipment and provide visual and audible indication that something needs to be, such as replacing a cylinder, or they may warn of any abnormal conditions that may require urgent action and a rapid response by staff. All alarm systems should be clearly labelled, and staff should know what to do if an alarm is triggered.

As part of these safety issues, checks must be undertaken - these might include daily ensuring that alarm panels, manifolds and visual indicators functioning and displaying correctly or that pressure gauges fare operating normally or that plant is not showing signs of overheating.

The other side of operating safety involves patient safety. Before any sedation, the patient and escort should be provided with specific instructions (verbally and in writing) about the possible effects of the proposed sedation and the various responsibilities both before and after treatment including fasting should be based on an individual assessment of the patient and the nature of the sedation and dental procedure.

The patient should be monitored perioperatively by a trained staff member in a manner suited to the patient and sedation technique and should be thoroughly assessed as fit for discharge. Depending on the patient's age, an adult escort - who can look after the patient unaided - should be present and accompany them home after treatment; however, adults receiving inhalation sedation with nitrous oxide/oxygen do not usually require an escort.

For further information visit https://bda. org/advice/Pages/Patient-care.aspx. https://doi.org/10.1038/s41404-020-0365-0 